\title{
Launch Vehicle Debris Models and Crew Vehicle Ascent Abort Risk
}

\author{
Ken Gee, NASA Ames Research Center \\ Scott L. Lawrence, PhD, NASA Ames Research Center
}

Keywords: Risk Analysis and Management, System Safety

\section{SUMMARY \& CONCLUSIONS}

In the event of a launch vehicle failure during ascent, a manned space launch system requires an ascent launch abort system that will be able to separate the crew module from the launch vehicle and return the crew safely to earth. One measure of the effectiveness and reliability of the launch abort system is its ability to reduce the risk of loss of crew from the failure environments, such as blast overpressure and debris strikes from an exploding launch vehicle, resulting from the launch vehicle failure scenario. Physics-based models are used to assess the evolution of, and risks presented by, the failure environments. In the case of debris strikes, the probability of the crew module being hit by at least one piece of debris is computed by modeling the evolution of the debris field over time and determining its relative position to the crew module. The characteristics of the debris field, including the number of pieces, the mass and reference area of each piece, the imparted velocity magnitude and direction and the ballistic coefficient, are defined by a debris catalog. A model has been created to generate a debris catalog using a combination of empirical- and physics-based models. The debris catalog model accounts for design features of the launch vehicle and the failure mechanisms involved in determining the number of pieces and imparted velocity. The model results are compared with a published catalog for the Space Shuttle external tank. The sensitivity of the risk prediction to the number of pieces and the imparted velocities are studied. The debris catalog generation model provides an additional tool in the risk assessment of ascent aborts for manned launch systems.

\section{INTRODUCTION}

For manned space launch systems, a reliable abort system is required to reduce the risks associated with launch vehicle failures during ascent. A typical launch abort system, shown in Figure 1, consists of a small, powerful rocket that carries the crew vehicle away from the launch vehicle in the event of an abort (Figure 2). During ascent (Figure 2(a)), any number of launch vehicle failure scenarios (e.g., engine failure, structural failure or control failure) can lead to activation of the launch abort system. The launch abort system separates the crew module from the launch vehicle (Figure 2(b)), creating sufficient separation distance to avoid the failure environments resulting from the destruction of the launch vehicle (Figure 2(c)). Failure environments include any hazards or conditions that can harm the crew, such as blast overpressure and debris, and are the end results of a failure scenario.

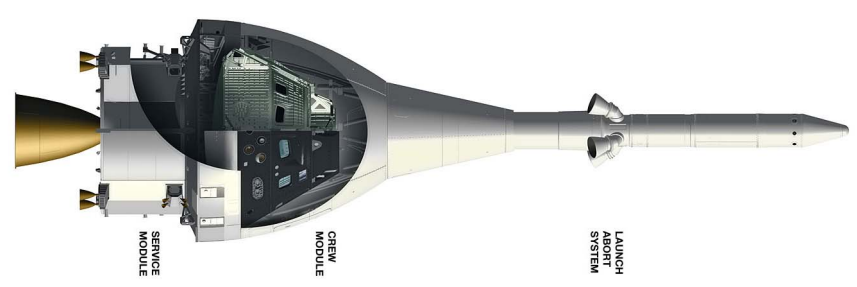

Figure 1. Launch abort system for NASA MPCV.

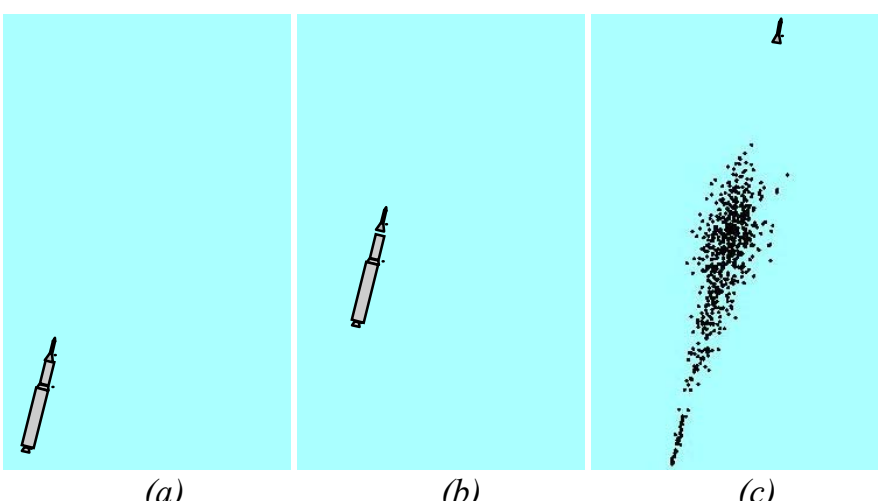

(a)

(b)

(c)

Figure 2. Ascent abort sequence with debris after launch vehicle destruct

The failure scenario starts with an initiating condition (e.g., leaks in propellant tanks) and progresses to the failure mechanism that creates the failure environments. If a failure scenario starts with leakage and leads to propellant mixing within a confined space, such as the intertank region, the failure mechanism is a confined-by-missile (CBM) explosion. For a failure scenario where leakage and mixing occur in an open space, the failure mechanism is a deflagration, resulting in a weaker shock wave. Physics-based blast models $[1,2]$ are used to predict the overpressure and impulse resulting from a CBM explosion or deflagration. Activation of the flight termination system (FTS) is another possible failure mechanism. In this case, debris is created by the linear shaped charge (LSC) and venting of the propellant. Failure scenarios 
involving loss of control can lead to structural failure of the launch vehicle due to aerodynamic loads. This failure scenario may not involve an explosion, but debris can be generated from the structural failure with velocities imparted by the venting propellant. All these mechanisms will cause the break-up of the launch vehicle, resulting in a debris field that poses a strike risk to the crew during an ascent abort.

The risk of the crew being struck by at least one piece of debris during an ascent abort can be predicted using a physicsbased model $[3,4]$ that computes the trajectory of the debris field and tracks the relative position of the debris and the crew module. This model requires as input a debris catalog, which describes each piece of debris in terms of its mass, reference area, aerodynamic characteristics and additional imparted velocity. A debris catalog is a function of the launch vehicle design, construction and the failure mechanism. Catalogs for specific vehicles and failure mechanisms are available, such as one developed for the Space Shuttle flight termination system by Hinckley et al. [5]. The Shuttle debris catalog was developed using detailed knowledge of the design and structure and was specific to one failure mechanism (activation of the flight termination system). For a risk and reliability analysis of an ascent abort system conducted early in the design cycle, when one ore more preliminary designs are being assessed, a faster, more general approach to generating a debris catalog is required. The approach must also be able to model the impact of different failure mechanisms on the overall risk. To meet this need, a more general physics-based debris catalog model has been developed.

The debris catalog model predicts the number of debris pieces and each debris piece mass, reference area and imparted velocity. The model accounts for launch vehicle parameters such as dry mass, propellant mass, and tank pressures. The imparted velocity is based on the failure mechanism, such as FTS activation, explosion or aerodynamic break-up. The following section describes the debris catalog model in more detail. The results of the model are used in the debris strike probability model, which provides an assessment of the risk the debris field poses to the crew module. Understanding the parameters that drive this risk can improve the safety and reliability of the launch abort system, crew module and launch vehicle.

\section{DEBRIS CATALOG MODEL}

The debris catalog model predicts the number of pieces, mass and imparted velocity of the individual pieces. The number of pieces and mass distribution are computed using a fragmentation model derived from studies on explosions. The imparted velocity is based on the failure mechanism involved. Velocities due to FTS activation are obtained from analysis of the propellant venting. Velocities due to explosions are obtained from solutions using a shock physics code.

Fragmentation of a structure due to an explosion has been extensively studied. Mott and Linfoot [6] proposed an exponential function relating the number of fragments greater than a given size to the total number of fragments, total mass and the average fragment mass. Sternberg [7] found that the formula held over the central part of the weight range but underpredicted the number of small pieces and overpredicted the number of large pieces. This shortcoming was addressed by dividing the fragment field into three regions based on debris mass, each with its own formula for predicting the mass distribution. The average mass of the fragments is used as the independent parameter for all the formulas. Mass is conserved. The Sternberg model is used to predict the mass distribution of the launch vehicle debris field.

The magnitude of the imparted velocity on a piece of debris is dependent upon the failure mechanism. For a CBM explosion, the imparted velocities are computed using CTH [8], a shock physics code. The volume of space available for propellant mixing and the amount and type of propellant are used to determine the amount of TNT that would result in the equivalent energy release [1,2]. The TNT material is placed in a model of the launch vehicle containing a representation of the tanks and walls. The Johnson-Cook fracture model and the Grady-Kipp fragmentation model are used to compute the fragmentation of the tanks and walls. The resulting CTH solution provides an imparted velocity profile as a function of axial location along the launch vehicle and an estimation of the relative number and size of the debris pieces. For imparted velocities due to an FTS event, the model developed by Hinckley et al. [5] is used. In this model, the linear shaped charge (LSC) is assumed to cut the propellant tank walls, allowing the propellant to vent due to internal pressure. Additional failure of the tank structure occurs due to stress concentrations in the cracks, creating debris pieces. The pieces are carried by the venting propellant, reaching maximum velocities based on the initial tank pressure and expected size of the holes. A similar approach is used to determine the imparted velocities due to a loss-of-control failure. In this case, structural failure due to aerodynamic loads lead to tank ruptures. These ruptures are expected to be larger than the initial cracks and holes created by the FTS event.

\section{DEBRIS CATALOGS}

The debris catalog model was used to create a catalog for the Space Shuttle external tank for comparison with the catalog developed by Hinckley et al. [6]. The average mass per debris piece was obtained from the Hinckley catalog. A comparison of the sorted debris mass distribution is shown in Figure 3. The catalog model predicted $17 \%$ fewer pieces than the Hinckley model and generally predicted larger pieces than the Hinckley model. The imparted velocity of each piece, as a function of the piece ballistic coefficient, is plotted in Figure 4. The model does not account for the copper sheathing of the LSC, which is the group of small, high speed $(\sim 800 \mathrm{~m} / \mathrm{sec})$ pieces in the Hinckley data. Otherwise, the imparted velocities compared well. The catalog model predicted a wider range of ballistic coefficients, but the values compared well with the Hinckley data. 


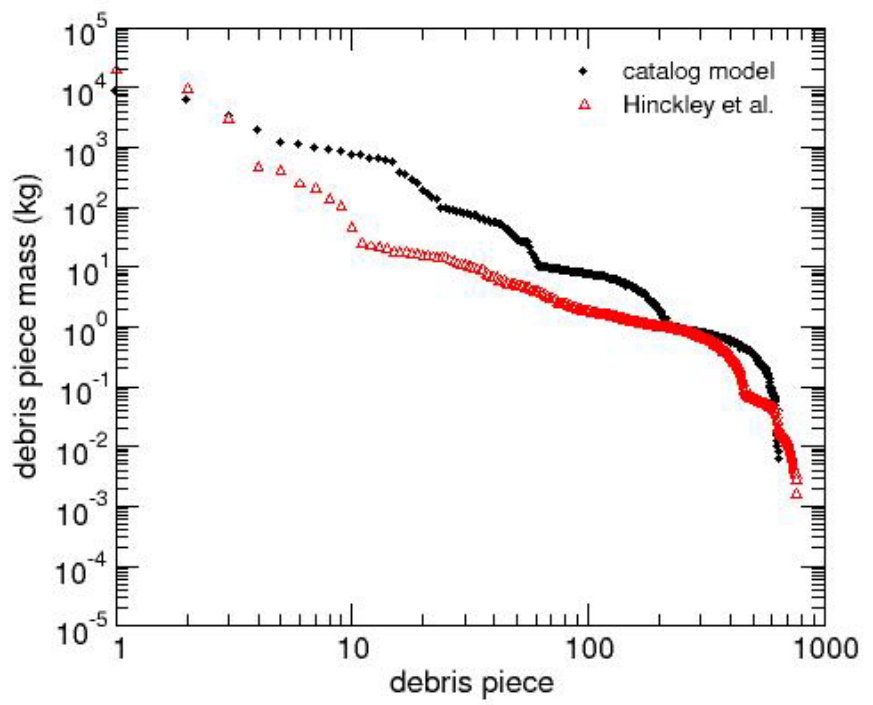

Figure 3. Comparison of debris mass distribution for Shuttle external tank

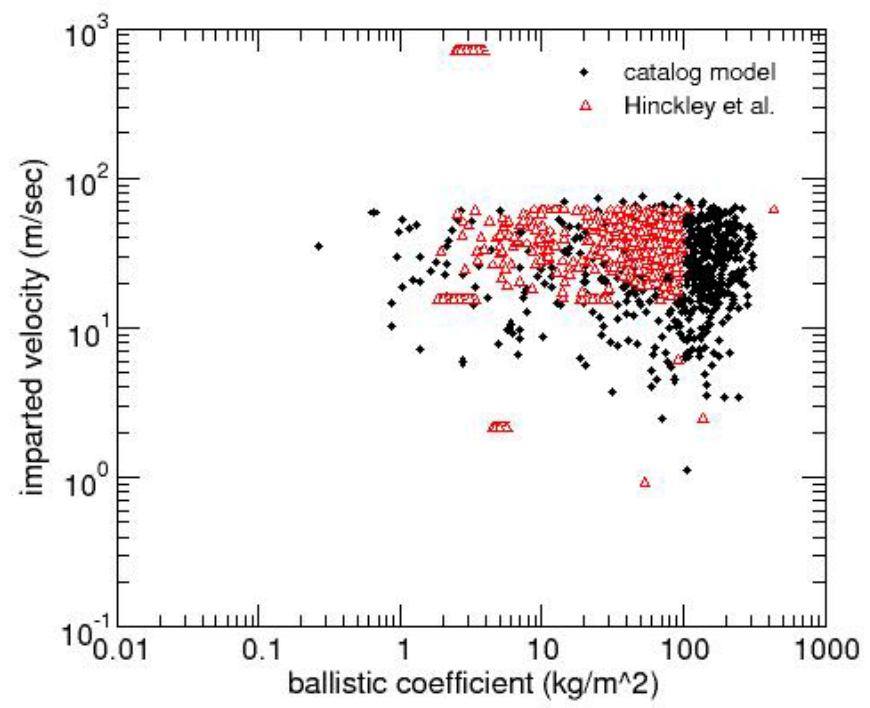

Figure 4. Comparison of imparted velocity for Shuttle external tank

From a risk perspective, the relevant measure is the probability of debris striking the crew module during an ascent abort. The strike probability is computed using the model described in References 7 and 8. Using a generic ascent trajectory and an MPCV-based crew module and launch abort system, the strike probabilities for aborts at various mission elapsed times (METs) are shown in Figure 5. Both catalogs produced similar strike probabilities. With zero warning time, the crew module is still on the launch vehicle at the time of destruct, resulting in a debris strike probability near 1.0. As the warning time increases, the debris strike probability decreases. With one second of warning time, the launch abort system is able to generate enough separation distance between the crew module and launch vehicle that the debris strike probability is near zero.

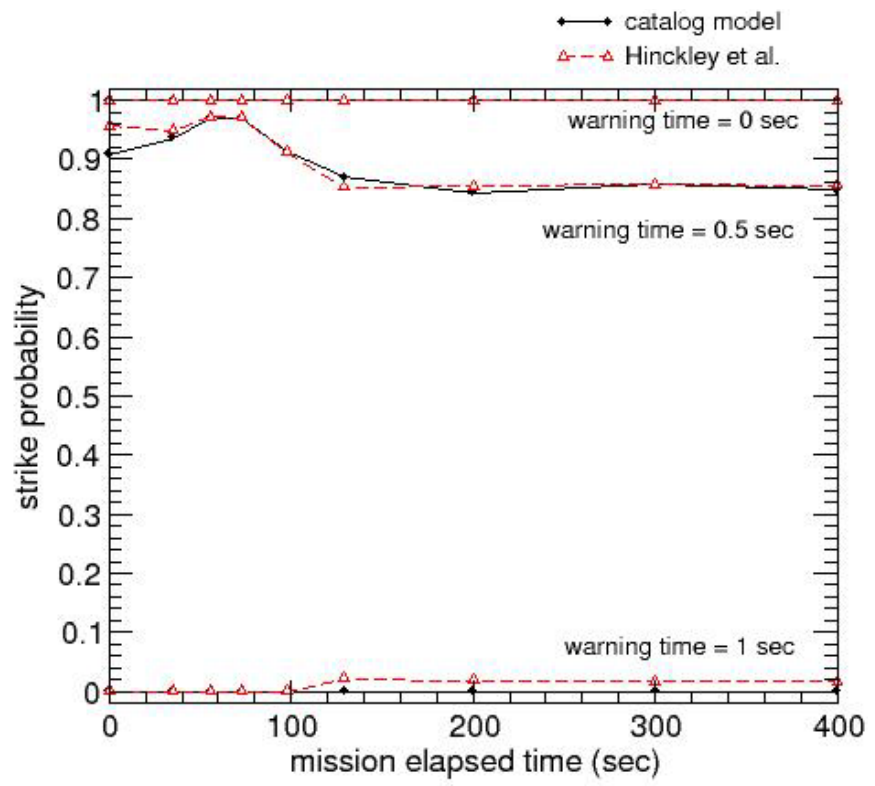

Figure 5. Debris strike probabilities of Shuttle external tank

Sensitivity studies were conducted to determine the effect of debris catalog parameters on the debris strike probability. Parameters of particular interest were the number of debris pieces and the imparted velocities. Within the debris catalog generation model, the number of pieces was controlled by the average mass, Mbar, of the debris pieces. Increasing the average mass decreased the number of pieces and vice versa, as shown in Figure 6. The Shuttle external tank debris field discussed above was used as the baseline. The effect of the number of pieces on the debris strike probabilities for aborts with $0.5 \mathrm{sec}$ warning time is shown in Figure 7. Debris strike probability exhibited a strong dependence on the number of debris pieces, with a reduction in risk corresponding to a reduction in the number of pieces.

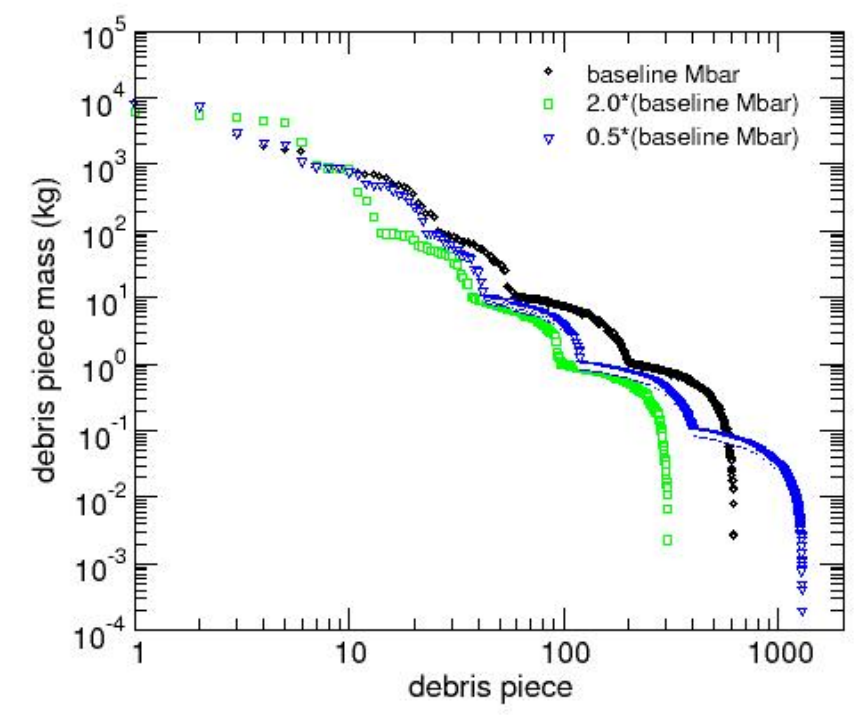

Figure 6. Debris mass distribution as function of Mbar 


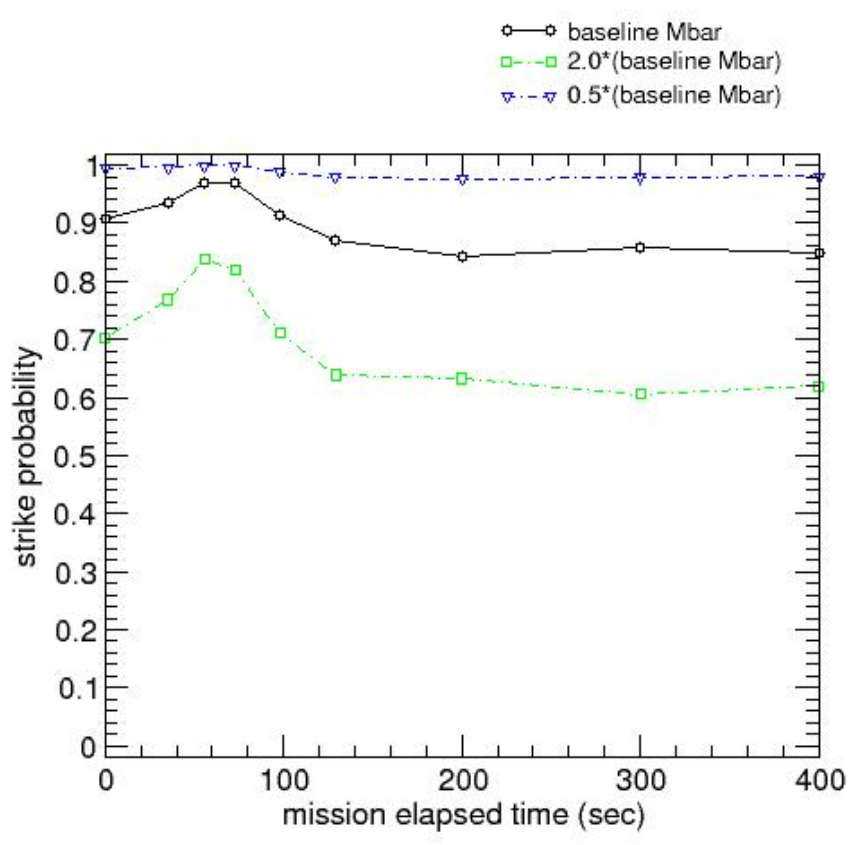

Figure 7. Effect of number of debris pieces on debris strike probability

Another parameter of interest was the imparted velocity on the debris. A failure involving an CBM explosion will generate debris with high imparted velocities near the center of the explosion. The size of the explosion will also impact the imparted velocities. Explosions resulting from larger equivalent TNT yields will create higher imparted velocities than explosions using smaller equivalent TNT yields. In the model, the imparted velocities from TNT explosions are computed using $\mathrm{CTH}$. A representative axisymmetric CTH model of the Shuttle external tank geometry with a TNT charge located in the intertank region is shown in Figure 8(a). A snapshot of the solution $0.01 \mathrm{sec}$ after detonation is shown in Figure 8(b). The imparted velocities as a function of axial location on the launch vehicle were obtained from the CTH solutions. The resulting maximum imparted velocity distributions due to explosions of various sizes, together with the velocity distribution from the FTS destruct failure mechanism, denoted as the baseline curve, are shown in Figure 9. The small explosive charge yielded a localized increase in velocity near the explosion center. As the explosive charge increased in size, the imparted velocities increased and affected a wider region of the launch vehicle. The velocity data are used in the debris catalog generation model to determine the imparted velocities of each debris piece in the Shuttle external tank debris field. The effect of velocity on debris strike probability for aborts with $0.5 \mathrm{sec}$ warning time is shown in Figure 10. In this case, the imparted velocities had little effect on the debris strike probabilities.

The debris catalog generation model presented in this paper is a physics-based tool developed for use in the engineering risk assessments of launch abort system for manned launch systems. Such risk assessments are conducted early in the design cycle, requiring a tool that can create debris catalogs based only on preliminary design information. The tool must also be able to account for the effects the failure mechanism has on the debris characteristics. To meet these requirements, the model uses an explosion-based model to predict the number of pieces and solutions from a shockphysics code to predict the imparted velocity distribution for failure mechanisms involving explosions. Imparted velocities due to venting of pressurized propellants are also modeled. The resulting debris catalog defines the number of debris pieces and the mass, size and imparted velocity of each piece. The debris catalogs are then used as input into a risk model used to predict the probability of the crew module being struck by at least one piece of debris during an ascent abort. The results of the model show good agreement with an existing debris catalog for the Shuttle external tank. Sensitivity analysis using the model indicate that strike probability is a strong function of the number of pieces in the debris catalog and a relatively weak function of the imparted velocity. The model provides additional capability to the Ames Engineering Risk Assessment tool set used to assess risks associated with manned space flight systems.

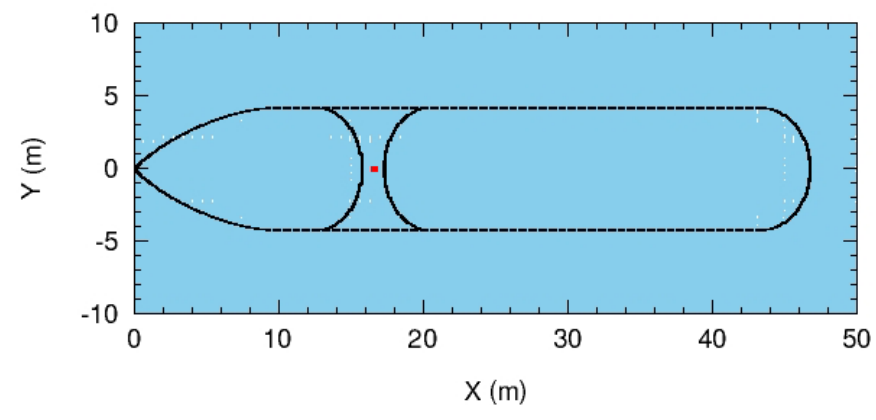

(a)

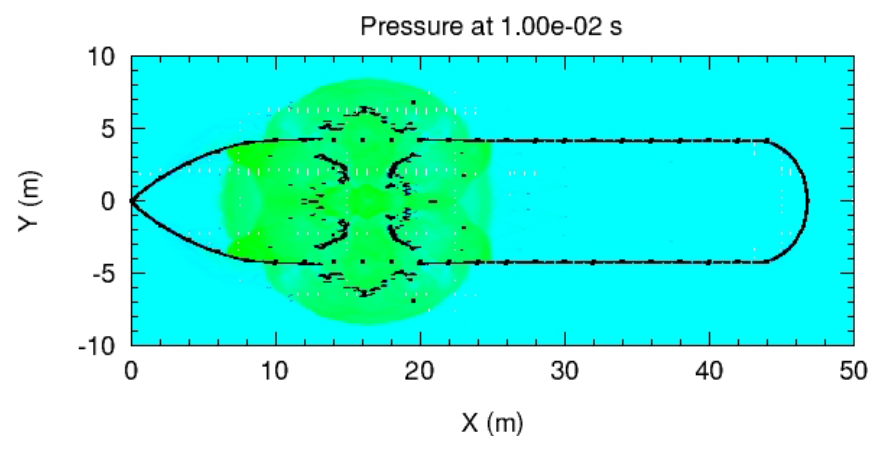

(b)

Figure 8. CTH model of geometry with TNT charge in intertank region 


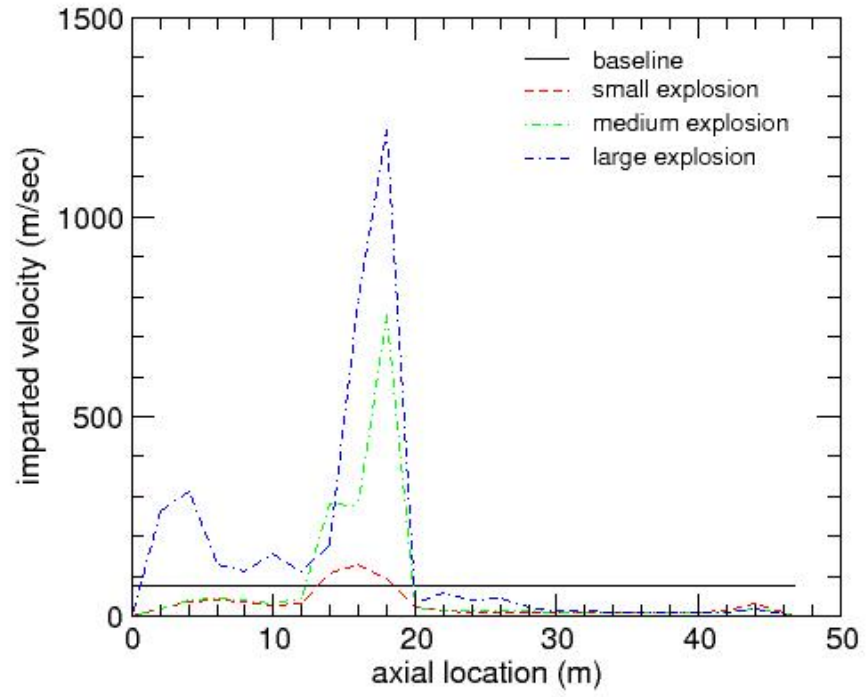

Figure 9. Imparted velocity as a function of axial location and size of TNT charge.

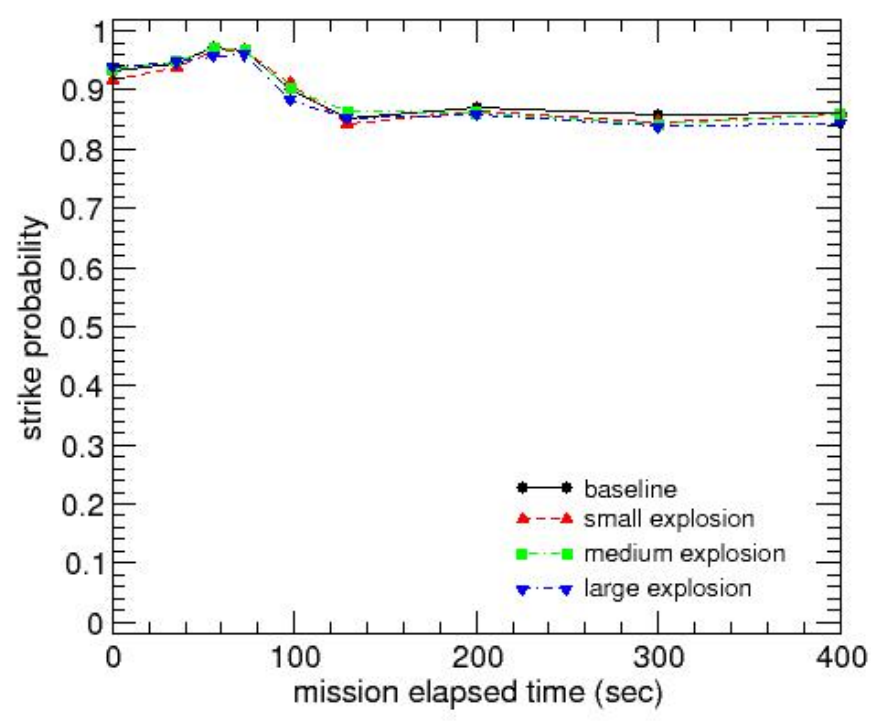

Figure 10. Effect of imparted velocity on strike probability

\section{REFERENCES}

1. Lawrence, S. L., Mathias, D., Gee, K., and Olsen, M., "Simulation Assisted Risk Assessment: Blast Overpressure Modeling," PSAM-0197, $8^{\text {th }}$ International Conference on Probabilistic Safety Assessment and Management (PSAM8), New Orleans, LA, May, 2006.

2. Lawrence, S. and Mathias, D., "Blast Overpressure Modeling Enhancements for Application to RiskInformed Design of Human Space Flight Launch Abort Systems," RAMS 06B-3, 2008 Reliability and Maintainability Symposium, Las Vegas, NV, January, 2008.

3. Gee, K. and Mathias, D., "Assessment of Launch Vehicle Debris Risk During Ascent Aborts," RAMSRM-312, The $54^{\text {th }}$ Annual Reliability and Maintainability Symposium,
Las Vegas, NV, January, 2008.

4. Gee, K. and Lawrence, S. L., "Sensitivity Analysis of Launch Vehicle Debris Risk Model," PSAM10 Probabilistic Safety Assessment and Management Conference, Seattle, WA, June, 2010.

5. Hinckley, W. M., Lehto, D. L., Coleburn, N. L., Gorechlad, A. J., Ward, J. M., and Petes, J., "Space Shuttle Range Safety Command Destruct System Analysis and Verification," NSWC TR-80-417, Naval Surface Weapons Center, Dahlgren, VA, 1981.

6. Mott, N. F. And Linfoot, E. H., "A Theory of Fragmentation," Ministry of Supply, A. C. 3348, January, 1943.

7. Sternberg, H. M., "Fragment Weight Distributions from Naturally Fragmenting Cylinders Loaded with Various Explosives," NOLTR 73-83, Naval Ordnance Laboratory, White Oaks, MD, October, 1973.

8. McGlaun, J. M., Thompson, S. L., Kmetyk, L. N., and Elrick, M. G., "CTH: A Three-Dimensional Shock Wave Physics Code,” Int. J. Impact Engng., 10:351, 1990.

\section{BIOGRAPHIES}

Ken Gee

MS 258-1

Ames Research Center

Moffett Field, CA 94035 USA

e-mail: Ken.Gee-1@,nasa.gov

Ken Gee is an aerospace engineer in the Systems Analysis Branch at NASA Ames Research Center. Prior to joining Ames, he was a senior research engineer with ELORET Corporation. He has over 20 years of experience in computational fluid dynamics, multidisciplinary analysis of flight vehicles and software development. He is currently a member of the Engineering Risk Assessment (ERA) project team at Ames. He has a Bachelor and Masters of Science degree from California Polytechnic State University, San Luis Obispo and an Engineer Degree from Stanford University.

Scott L. Lawrence

MS 258-1

Ames Research Center

Moffett Field, CA 94035 USA

e-mail: Scott.L.Lawrence@nasa.gov

Scott Lawrence is an Aerospace Engineer in the Systems Analysis Branch at NASA Ames Research Center. During his 26 years at NASA, Dr. Lawrence has worked to develop and apply computational fluid dynamics methods to problems in hypersonic aerothermodynamics, supersonic aerodynamics, as well as crew safety. His recent work has focused on applying analysis methods of various levels of fidelity to the characterization of failure environments created by catastrophic failure of a launch vehicle. Dr. Lawrence received his B.S., M.S., and PhD. degrees in Aerospace Engineering from Iowa State University, in Ames, Iowa. 